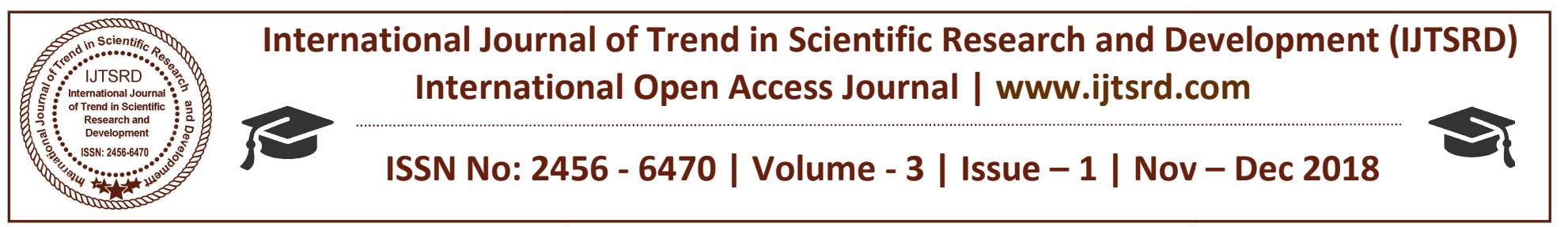

\title{
Review of Electro Chemical Machining
}

\author{
R. Karimdadashi \\ Department of Mechanical Engineering, Faculty of Engineering, University of Bonab, Bonab, Iran
}

\begin{abstract}
Electro-Chemical Machining (ECM) is the generic term for a variety of electro-chemical processes. ECM is used to machine work pieces through the anodic dissolution of metal. The process is used in aerospace engineering and the automotive, construction, medical equipment, micro system and power supply industries. Almost all kinds of metal can be electro-chemically machined, especially high-alloyed nickel- or titaniumbased ones, as well as hardened materials.
\end{abstract}

As it is a contactless procedure with no heat input, the process is not subject to any of the disadvantages experienced with traditional machining methods, e.g. tool wear, mechanical stresses, micro-fissures caused by heat transfer or the need for subsequent deburring operations. All electro-chemical machining processes are characterized by stress-free stock removal, gentle transitions and top-quality surfaces without burr formation.

Key Words: Electro chemical machining, Anodic, Contactless, Traditional machining.

\section{INTRODUCTION}

Electrochemical machining (ECM) is a method of removing metal by an electrochemical process. It is normally used for mass production and is used for working extremely hard materials or materials that are difficult to machine using conventional methods.[1] Its use is limited to electrically conductive materials. ECM can cut small or odd-shaped angles, intricate contours or cavities in hard and exotic metals, such as titanium aluminides, Inconel, Waspaloy, and high nickel, cobalt, and rhenium alloys.[2] Both external and internal geometries can be machined.

ECM is often characterized as "reverse electroplating", in that it removes material instead of adding it.[2] It is similar in concept to electrical discharge machining (EDM) in that a high current is

passed between an electrode and the part, through an electrolytic material removal process having a negatively charged electrode (cathode), a conductive fluid (electrolyte), and a conductive workpiece (anode); however, in ECM there is no tool wear.[1] The ECM cutting tool is guided along the desired path close to the work but without touching the piece. Unlike EDM, however, no sparks are created. High metal removal rates are possible with ECM, with no thermal or mechanical stresses being transferred to the part, and mirror surface finishes can be achieved.

In the ECM process, a cathode (tool) is advanced into an anode (workpiece). The pressurized electrolyte is injected at a set temperature to the area being cut. The feed rate is the same as the rate of "liquefaction" of the material. The gap between the tool and the workpiece varies within $80-800$ micrometers $(0.003-$ 0.030 in.)[1] As electrons cross the gap, material from the workpiece is dissolved, as the tool forms the desired shape in the workpiece. The electrolytic fluid carries away the metal hydroxide formed in the process.[2]

As far back as 1929, an experimental ECM process was developed by W.Gussef, although it was 1959 before a commercial process was established by the Anocut Engineering Company. B.R. and J.I. Lazarenko are also credited with proposing the use of electrolysis for metal removal.[2]

Much research was done in the 1960s and 1970s, particularly in the gas turbine industry. The rise of EDM in the same period slowed ECM research in the west, although work continued behind the Iron Curtain. The original problems of poor dimensional accuracy and environmentally polluting waste have largely been overcome, although the process remains a niche technique. 
The ECM process is most widely used to produce complicated shapes such as turbine blades with good surface finish in difficult to machine materials. It is also widely and effectively used as a deburring process.[2]

In deburring, ECM removes metal projections left from the machining process, and so dulls sharp edges. This process is fast and often more convenient than the conventional methods of deburring by hand or nontraditional machining processes.[1]

ECM process can be more economical if a conductive wire is used as a tool since it helps to prevent tool profiling. Using wire-tool allows cutting complex shapes with no need for large amount of power supplies. Using wire-tool in ECM is known as Wire Electrochemical Machining (WECM).[3]

\section{PRINCIPAL OF ECM}

ECM is the controlled removal of metal by anodic dissolution in an electrolytic cell in which the work piece is the anode and the tool is cathode [4]. Electrochemical machining is developed on the principle of Faradays and $\mathrm{Ohm}$. In this process, an electrolyte cell is formed by the anode (work-piece) and the cathode (tool) in the midst of a following electrolyte. The metal is removed by the controlled dissolution of the anode according to the well-known Faradays law of electrolysis. Fig1.Shows two electrodes which are placed closely with a gap of about $0.5 \mathrm{~mm}$ and immersed in an electrolyte which is a solution of sodium chloride (common salt).

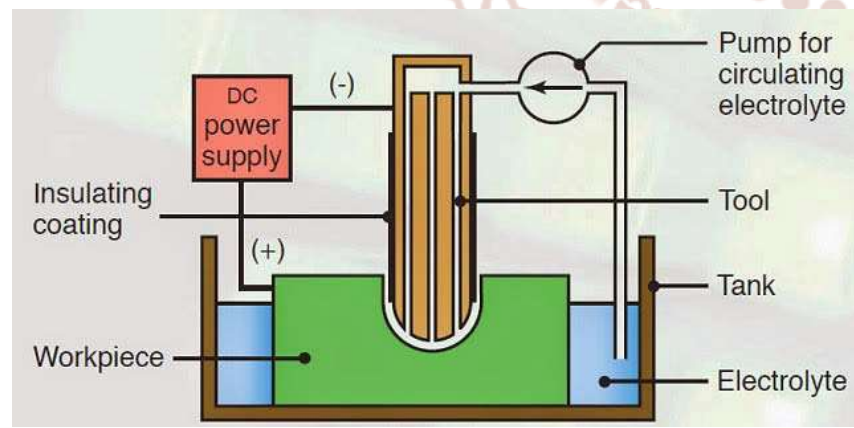

Fig 1: schematic for electrochemical machining process

The high current densities promote rapid generation of metal hydroxides and gas bubble in the small spacing between the electrodes. These become a barrier to the electrolyzing current after a few seconds. To maintain a continuous high density current, these products of machining must be continuously removed. This is achieved by circulating the electrolyte at a high velocity through the gap between the electrodes. It is also to be noted that the machining gap size increases as the metal is removed. The larger gap leads to a decrease in the metal removal rateTherefore to maintain a constant gap between the tool and work-piece, the cathode (tool) should be advanced towards the anode (work) at the same rate at which the metal is removed

\section{ELECTRO CHEMICAL MACHINING OF STEEL}

In ECM, emphasize should be given to the machining process parameters, to achieve the desired responses or performance characteristics. The variables in the machining have to be chosen wisely and not by random samples by conducting the experiments based on Taguchi techniques, thereby reducing the costs incurred in tooling and operating cost. Hocheng et al [4] proposed that by increasing the values of the electrical voltage, electrolyte concentration, time of electrolysis and inter electrode gap (IEG) in ECM, the MRR increases for SKD61 SS using sodium nitrate as the electrolyte and a flat end copper electrode. Neto et al [5] have studied ECM by varying four process parameters like feed rate, electrolyte, flow rate of the electrolyte and the voltage in the valve steel and found the better responses on MRR, surface roughness $(\mathrm{Ra})$ and overcut by using two electrolytes sodium chloride $(\mathrm{NaCl})$ and sodium nitrate $(\mathrm{NaNO} 3)$ and concluded that the feed rate was the important parameter which affects the response MRR significantly. Uttarwar et al [6] have analyzed the result of ECM by varying the voltage parameter in AISI 302B stainless steel (SS) and obtained the better results in MRR. Bhattacharya et al [7] have employed the response surface methodology (RSM) as the optimization technique and studied the variables for maximizing the responses like MRR and also to minimize the overcut while doing electrochemical machining processes in EN19 tool steel. Asokan et al [8] have suggested the multi objective optimization by varying the parameters like current, voltage, feed rate and gap between the electrode and the anode and have come up with the improved MRR and Ra by utilizing multi regression models and artificial neural networks (ANN). Chakradhar et al [9] employed Taguchi technique and a multi objective technique grey relational analysis to determine the optimal process parameters like voltage, feed and electrolyte concentration in ECM of EN31 tool steel. They concluded that the feed rate affects the MRR 
significantly, therey by reducing the overcut, cylindricity error and the Ra. Bisht et al [10] have taken four parameters and analyzed the voltage, electrolyte flow rate, tool feed rate and current and they have used single optimization technique to optimize their responses MRR and surface roughness in both work piece materials, namely aluminum and mild steel. They concluded that the electrolyte flow rate was the significant parameter for aluminum and voltage for mild steel to increase the MRR and to decrease the surface roughness. Ghoreishi et al [11] have employed EDM for machining tungsten carbide cobalt composite (WC-Co) and optimized the process parameters using face centered central (FCC) composite design using response surface methodology (RSM). They have also concluded that the variables like current, pulse on time, duty cycle and gap voltage affects the responses like MRR and tool wear rate (TWR) and the interactions between the current with pulse on time and gap voltage with current affects the Ra significantly. Das et al [12] have implemented artificial bee colony algorithm for studying the electrochemical machining of EN31 tool steel to optimize the responses. They have employed scanning electron microscopy (SEM) images for investigating the work piece material surfaces and have concluded that the electrolyte concentration plays an important role in minimizing the SR. Habib [13] have used the parameters like current, voltage, electrolyte concentration and tool feed rate and optimized the variables using single objective optimization technique and concluded that the current affects the SR and the voltage increases the MRR significantly. Barman et al [14] have investigated ECM variables on MRR and different attributes of Ra in EN31 tool steel using multi-response optimization techniques and they have concluded that the electrolyte concentration have an important effect on the output responses. Rao et al [15] investigated the electro chemical machining using Taguchi method. They have done many trials by altering the variables like voltage, electrolyte concentration, feed rate and percentage of reinforcement to maximize the material removal rate and to minimize the surface roughness and radial over cut (ROC). They have also used analysis of variance (ANOVA) table to conclude that the feed rate of the cathode tool was the most significant parameter for obtaining the optimized responses. This work was aimed to investigate the material removal rate of SKD12 tool steel and also to examine the effect of process parameters like current, voltage and electrolyte concentration on maximizing the material removal rate (MRR).

\section{ELECTROLYTE IN THE ELECTRO CHEMICAL MACHINING}

The electrolyte in the processes of Electro Chemical Machining has several functions: the current is carried between the tool and the workpiece through electrolyte, the produced heat is dissipated by the liquid electrolytic solution, the product of machining is removed by the solution and it keeps the reactions continuous by supplying the elements necessary for the reaction. For different types of machined materials different types of electrolytes are used in Electro Chemical Machining: -Sodium chloride $(\mathrm{NaCl})$ at the concentration of $20 \%$ - for ferrous alloys (e.g. Steels and cast irons and cobalt alloys).

Sodium nitrate (NaNO3) - for ferrous alloys.

Hydrochloric acid $(\mathrm{HCl})$ - for nickel alloys.

A mixture of sodium chloride $(\mathrm{NaCl})$ and sulfuric acid (H2SO4) - for nickel alloys.

A mixture of $10 \%$ hydrofluoric acid (HF), $10 \%$ hydrochloric acid $(\mathrm{HCl}), 10 \%$ nitric acid

(HNO3) - For titanium alloys.

Sodium hydroxide $(\mathrm{NaOH})$ - for tungsten carbide (WC).

The selection of the electrolyte should be done by considering the following matters: required Machining rate, required dimensional accuracy and surface texture and integrity.

The electrolyte should possess several important properties

$>$ Electrolyte must possess high electrical conductivity.

Electrolyte should possess high specific heat.

The viscosity must be as low as possible.

The electrolyte must be chemically stable.

Electrolyte must be chemically active to cause the better metal or material removal rate.

$>$ Electrolyte should not form any type of excess layer on the top of electrolyte, tool or the work piece.

$>$ Electrolyte should not be toxic and corrosive.

$>$ Electrolyte should be economical and easily available.

Perfect electrolyte flow across the machining tool is mandatory for proper machining. Cavitation is likely to be occurred in the tool. So proper care is necessary to keep the tool in shape. Tool design must ensure the uniform flow of electrolytic solution in all machining 
areas. Optimum flow of the electrolyte is desired because excessive flow can cause erosion of the tool. Mainly two types of flows are used: divergent flow and convergent flow. Convergent flow provides a smoother flow of electrolytes. The advantages of convergent flow system are:

$>$ improved surface finish

$>$ improved uniform and predictable side over cut as well as front machining gap,

$>$ less prone to arcing,

$>$ clean operating environment and

$>$ stray currents make it possible to be eliminated unwanted machining.

But it is also important to be mentioned that machining

\section{EQUIPMENT OF ECM}

$>$ DC Power supply. The machining rate in electrochemical machining is proportional to the electric current density. In order to achieve high values of the machining rate electrochemical machining is commonly performed at a high direct current exceeding $1000 \mathrm{~A}$. The gap between the tool and the work piece must be low for highpitched correctness, thus the voltage must be small to prevent a short circuit. The voltage of the process is $5-25 \mathrm{~V}$. The control system uses some of this electrical power.

$>$ Electrolyte circulation system. The products of the electrochemical reaction should be removed from the gap between the work piece and the tool. Accumulation of the reaction products causes decrease of the process efficiency and reduction of the rate of machining. Therefore the electrolyte flow speed should be high. Commonly it is in the range $300-3,000 \mathrm{~m} / \mathrm{min}(1,000-10,000 \mathrm{ft} / \mathrm{min})$. The inlet pressure should be in between $0.15-3$ $\mathrm{MPa}$. The electrolyte system should comprise a fairly strong pump. The electrolyte is continuously filtered in order to trap the precipitated reaction products (sludge).

$>$ Control system. Electrical parameters of the process (voltage and current)), tool feed speed and parameters of electrolyte circulation system (inlet and outlet pressure of electrolyte, temperature of electrolyte) are controlled by the control system, which provide stable and efficient operation of the unit

$>$ Mechanical system. It consist of the table, the frame, work enclosure (prevents the electrolyte from spilling), the work head (where the tool is
mounted).The tools (electrodes) are also part of the mechanical system. One of the most important parameters of electrochemical machining is maintaining a constant voltage level. This is achieved by the control system providing a movement of the tool at a constant speed equal to the linear rate of machining. The process in a steady state is performed at a constant (typically gap 0.1-0.4 mm / 0.004-0.016"). A firm fixation of the work piece provided by the fixture, the table and the frame is also important for stable operation of the system at a constant gap. Conventional machining equipment including CNC machines may be modified for electrochemical machining process.

\section{APPLICATIONS OF ELECTRO CHEMICAL MACHINING}

Electro Chemical Machining is mainly used in the areas where conventional machining techniques are not feasible [7]. Theoretically Electro Chemical Machining could be used for machining the all electro conductive metals and alloys. The usual applications of Electro Chemical Machining are:

$>$ Machining of hard materials and heat resistant materials. The process parameters and the tool life time do not depend on the hardness of the work piece. Therefore electrochemical machining is often used for machining hard materials.

Machining of cavities in forging dies, drilling deeper holes and irregular shaped holes which cannot be obtained by conventional machining methods.

Machining of complex profiles like turbine wheels, turbine and jet blades.

Die sinking. Electro Chemical Machining is often used as an alternative to the cavity type electric discharge machining (EDM).

Fabrication of thin walled parts. Electrochemical machining does not produce surface stress in the work piece therefore even very brittle and easily deformed materials may be machined in thin walled shapes.

$>$ Grinding of a work piece by a rotating wheel, which performs grinding operation through an electrolyte. The wheel is conductive and catholically connected. Non-conductive hard particles are set on the wheel surface. The particles provide a constant gap through which an electrolyte is continuously fed. Hard and brittle materials are ground by the method. 
$>$ Rough corners or edges can be turned into very smooth parts and the process is known as deburring.

\section{MATERIALS USED IN EXTREME CONDITION}

With Electro Chemical Machining is possible processing of practically all groups of constructional steels and alloys used in the industry, including highstrength steels and alloys, metal-ceramics, nanostructure alloys. This materials could be easily machined by Electro Chemical Machining [8]: alloy steels and construction steels, corrosive resistant steels, high-carbon chromium steels, stainless chromenickel steels, tool steels, chromium-nickel heatresistant alloys, HSS, copper and copper alloys, nickel and nickel alloys, magnets, titanium and its alloys, ceramic-metal hard alloys (WC-Co, WC-TiC-Co), nanostructure steels and alloys. Most of these materials with exception of copper, copper alloys and magnets could be used in extreme conditions of temperature, loading, friction, and wear, corrosion, in Energy, Transportation and Machinery manufacturing industries.

Electro Chemical Machining has a significant place among the methods of machining of conducting materials. It is applied in cases where alternative methods (especially the traditional ones) encounter problems: for operations which are time and handlabor consuming. Electro Chemical Machining is feasible for the machining of difficult-to-cut materials (high-strength, tough, hard, and brittle alloys, superalloys), complex-shaped parts, and the machining in the difficult-to-reach areas. In some cases, Electro Chemical Machining enables one to perform unique operations, for example, drilling of very small, deep, curved holes. The Electro Chemical Machining processes are in progress, spread into new areas of modern technologies, for instance, micromachining. Electro Chemical Machining today is widely used in processing of materials used in extreme conditions of temperature, loading, friction, wear, corrosion, in Energy, Transportation and Machinery manufacturing industries.

Surface roughness is an important measurement in chemical machining. It affects the mechanical properties (friction and wear) of substrates and therefore, it would have significant impact on substrate application, notably on the substrate bonding. The mechanism of direct substrate bonding at room temperature has been attributed to the short range intermolecular and interatomic attraction forces, such as Van de Waals forces. High surface roughness will result in small real area of contact, and therefore yield voids at the bonding interface. When the surface roughness exceeds a critical value, the substrates will not bond at all. In this study, surface roughness ( $\mathrm{Ra})$ was measured in nm by AFM before and after the $\mathrm{CHM}$ process. The measurement assessed the improvement of surface roughness after the chemical machining process. Thus, the value obtained either is negative (the surface roughness getting poorer after chemical machining) or positive (the surface roughness getting better after the chemical machining). Consequently, the higher the positive value of surface roughness, the better it is. Analytical study of surface roughness is similar to etch rate, where its first and second order models were developed with DE 7. This is followed by surface roughness studies, where the relationships between surface roughness and variables such as etching temperature was assessed as well as their influence on the surface roughness was investigated.

\section{MACHIN ABLE GLASS CERAMICS}

The first practical glass ceramics were developed nearly fifty years ago. These glass ceramics are prepared by the controlled crystallization of special glasses. The original glass ceramics were produced by inducing volume nucleation in melt-derived bulk silicate glasses, usually by the addition of nucleating agents. Since that time, a wide variety of applications of these versatile materials have been developed as a result of their many outstanding properties and the distinct advantages of the glass ceramic method, in certain circumstances, over conventional ceramics processing routes.

More recently, glass ceramic processing has been greatly extended to include no silicates and even nonoxide compositions, and also the preparation of the precursor glasses by sol-gel techniques. Ordinary glass is non-crystalline. Glass ceramics however, are polycrystalline materials that are manufactured by controlled crystallization of suitable base glasses Glass ceramics represent an extreme case because the material, originally a glass, is deliberately heat treated to transform it into a new material whose polycrystalline structure gives rise to totally different set of properties. Because of these dependencies, glass properties are listed for glasses in the annealed state, and the properties of glass-ceramics are given on the 
basis of the manufacturer's standard production process.

Glass ceramics are mechanically strong material and can sustain repeated and quick temperature change up to $800-1000 \mathrm{oC}$. At the same time, they have very low heat conduction coefficient and can be nearly transparent for radiation in the infrared wavelengths. Chemical durability of glass ceramics is strongly dependent upon their composition. In addition, previous thermal history and mode of forming can have pronounced effects. For glass ceramics, the amount and composition of the crystalline and glass phases, as well as the microstructure, have effects that may well substantially alter the intrinsic durability expected on the basis of the overall bulk composition.

Glass ceramics are useful in thermally hazardous conditions. This glassy material contains crystalline lattices, which give the distinct properties of glass ceramics. Glass ceramics possess good resistance to erosion and pressure, as well as excellent hardness, and make them widely used in industrial purposes. Moreover, glass ceramics are very good electrical insulator.

Research works done on machining of Machin able glass ceramics by chemical etching is limited due to their resistance to the chemical effects, and they have become the last material to be used in chemical etching. Dry etching of glass had been reported by using SF6. Watanabe obtained a linear relationship between etch rate and temperature while compared between wet etching and mechanic machining.

This was probably caused by the limited chemical reaction.

Period and reported that the obtained etch rate was surprisingly linear. They concluded that a minor change of etching time had slightly changed the linear shape of etch rate to curvature; and Olsen et al. also indicated that increase in etching time would decrease the bond strength by increasing etch rate.

\section{CONCULUSION}

From the results obtained we are able to conclude that etch rate was influenced by etching temperature and etchant used for the selected materials. In MGC, etch rate was also influenced by the etching duration. For $\mathrm{SiC}$, the etch rate shows dependency on the on both the etching duration and individual etchant involved. Results also showed that etch rate increased with the increase of etching temperature for $\mathrm{MGC}$ and $\mathrm{BN}$ with exception of $\mathrm{SiC}$ was obtained. $\mathrm{MGC}$ and $\mathrm{BN}$ show the best result while etching in $\mathrm{HBr}$ etchant; and, $\mathrm{SiC}^{\prime}$ s best etch rate occurred in $\mathrm{H} 3 \mathrm{PO} 4$ etchant. The difference etch rate was observed for the substrates suggests that the reaction mechanism involved for the individual etching process could be different, however further kinetic data are required to support this notion. Additionally, the findings confirm that selection of the right etchant for specific substrate is important in achieving the best etching result. Can affect the difference between these two.

\section{REFERENCES}

1. Todd, H. Robert; Allen, K. Dell; Alting, Leo (1994), Manufacturing Processes Reference Guide (1st ed.), Industrial Press Inc., pp. 198-199

2. Valenti, Michael, "Making the Cut." Mechanical Engineering, American Society of Mechanical Engineers, 2001

3. Ahmed Maged, Salah Haridy, Mohammad Shamsuzzaman, Imad Alsyouf, and Roubi Zaied

4. Hocheng, H., Sun, Y. H., Lin, S. C., \& Kao, P. S., "A material removal analysis of electrochemical machining using flat-end cathode", Journal of Materials Processing Technology, Vol. 140(1), 264-268, 2003

5. Neto. J, Silva. E, and Silva. M, "Intervening variables in electrochemical machining", Journal of materials processing technology, Vol. 179, pp. 92-96, 2006

6. Uttarwar, S., and Chopade, I., "Effect of voltage variation on MRR for stainless steel EN series 58A (AISI 302b) in electrochemical machining: a practical approach" Proceedings of the World Congress on Engineering, Vol. II, London, U.K., 2006

7. Bhattacharya, B. and Sorkhel, P.B., "Investigation for controlled electrochemical machining through response surface methodology-based approach", Journal of Materials Processing Technology, Vol. 86, pp. 200-207, 2006

8. P. Asokan \& R. Ravi Kumar, R. Jeyapaul, M. Santhi, "Development of multi-objective optimization models for electrochemical machining process", International Journal of Advanced Manufacturing Technology., Vol. 39, pp. 55-63, 2008 
9. Chakradhar, D., and Gopal, A., "Multi-Objective Optimization of Electrochemical machining of EN31 steel by Grey Relational Analysis", International Journal of Modeling and Optimization, Vol. 1, No. 2, pp. 113 - 117, 2011

10. Bisht, B., Vimal, J., and Chaturvedi, V., "Parametric Optimization of Electrochemical Machining Using Signal-To-Noise (S/N) Ratio", International Journal of Modern Engineering Research, Vol. 3, Issue.4, pp. 1999-2006, 2013

11. Assarzadeh S, Ghoreishi M, "Statistical Investigation into the Effects of Electro-Discharge Machining Parameters on $\mathrm{WC} / 6 \%$ Co CompositePart 1: Modeling through Response Surface Methodology (RSM)", Advanced materials manufacturing and characterization, Vol. 3, Issue. 2, pp. $478-486,2013$

12. Das, M., Kumar, K., Barman, T., and Sahoo, P., "Investigation on Electrochemical Machining of EN31 Steel for Optimization of MRR and Surface
Roughness using Artificial Bee Colony Algorithm", Procedia Engineering, Vol. 971, pp. $1587-1596,2014$

13. Habib, S., "Experimental Investigation of Electrochemical Machining Process using Taguchi Approach", International Journal of Scientific Research in Chemical Engineering, Vol. 1(6), pp. 93-105, 2014

14. Rao, S., and Padmanabhan, G., "Parametric optimization in electrochemical machining using utility based taguchi method", Journal of Engineering Science and Technology, Vol. 10, No. 1, pp. 81 - 96, 2015

15. McGeough J.A., Principles of Electro Chemical Machining, Chapman and Hall, London 1974

16. Ross P.J., Taguchi techniques for quality engineering, McGraw-Hill, New York, 1988

17. G. Taguchi, Introduction to Quality Engineering, Asian Productivity Organization, Tokyo, 1990. 